\title{
Regional Heart Motion Abnormality Detection via Multiview Fusion
}

\author{
Kumaradevan Punithakumar ${ }^{1}$, Ismail Ben Ayed ${ }^{1}$, Ali Islam², \\ Aashish Goela ${ }^{3}$, and Shuo $\mathrm{Li}^{1}$ \\ 1 GE Healthcare, London, Ontario, Canada \\ 2 St. Joseph's Health Care, London, Ontario, Canada \\ 3 London Health Sciences Centre, London, Ontario, Canada \\ \{Kumaradevan.Punithakumar, Ismail.BenAyed, Shuo.Li\}@ge.com, \\ Aashish.Goela@lhsc.on.ca, Ali.Islam@sjhc.london.on.ca
}

\begin{abstract}
This study investigates regional heart motion abnormality detection via multiview fusion in cine cardiac MR images. In contrast to previous methods which rely only on short-axis image sequences, the proposed approach exploits the information from several other long-axis image sequences, namely, 2-chamber, 3-chamber and 4-chamber MR images. Our analysis follows the standard issued by American Heart Association to identify 17 standardized left ventricular segments. The proposed method first computes an initial sequence of corresponding myocardial points using a nonrigid image registration algorithm within each sequence. Then, these points were mapped to 3D space and tracked using Unscented Kalman Filter (UKS). We propose a maximum likelihood based track-to-track fusion approach to combine UKS tracks from multiple image views. Finally, we use a Shannon's differential entropy of distributions of potential classifiers obtained from multiview fusion estimates, and a naive Bayes classifier algorithm to automatically detect abnormal functional regions of the myocardium. We proved the benefits of the proposed method by comparing the classification results with and without fusion over 480 regional myocardial segments obtained from 30 subjects. The evaluations in comparisons to the ground truth classifications by radiologists showed that the proposed fusion yielded an area-under-the-curve (AUC) of 95.9\%, bringing a significant improvement of $3.8 \%$ in comparisons to previous methods that use only short-axis images.
\end{abstract}

\section{Introduction}

Accurate detection of motion abnormality of regional myocardial segments in MRI is essential in the diagnosis and treatment of coronary heart disease [71115], the leading cause of death worldwide. The problem has attracted a recent research attention recently [7115]. Unfortunately, existing MRI-based methods rely only on short-axis sequences [711:15. However, the actual LV motion is a complicated combination of motions in 3D space. Little or no through-plane motion information is available from standard single view $2 \mathrm{D}$ sequences, which 
severely limits the accuracy of 3D motion estimation. Therefore, exploiting information from other orthogonal image sequences can lead to a more accurate assessment of cardiac motion.

The purpose of our study is to develop a regional heart motion abnormality detection algorithm via multiview fusion, thereby exploiting the information from both short- and long-axis MRI sequences, namely, 2-chamber, 3-chamber and 4-chamber images. The proposed algorithm is based on a novel, multiview 3D motion estimation technique which consists of two main components, preprocessing and track-to-track fusion, both depicted in Fig. 1(a). Our 3D motion estimation is fundamentally different from existing 3D motion estimation methods, e.g., those based on incompressible models [2, deformable models [514, $3 \mathrm{D}$ harmonic phase [10] or short- and long-axis image registration 9, among others. Furthermore, it uses only standard clinical data (i.e., cine MRI 1 ), unlike most of existing methods which either use data that are not available in regular clinical routine, such as displacement encoding with stimulated echoes (DENSE) MR images 14, or data that increase the scan time, such as myocardial tagging [510].

The proposed method first computes an initial sequence of corresponding myocardial points using a nonrigid image registration algorithm [4] within each $2 \mathrm{D}$ sequence, long- and short-axis, given a user-provided segmentation of the first frame. In order to provide a temporal smoothing to the dataset, we used a 3D extension of the nonlinear state transition model in [11. Then, we propose to use an unscented Kalman smoother (UKS), a recursive nonlinear Bayesian approach, to obtain the state estimates and the corresponding covariance estimates. The state vector consists of position and velocity information of endo- and epi-cardial points over a cardiac cycle.

The main contributions of this study is a track-to-track multiview fusion based on a maximum-likelihood formulation which combines the UKS estimates from different views from short- and long-axis image sequences, thereby obtaining accurate 3D motion estimates. Track-to-track fusion problems are common in the multisensor fusion literature [1] but, to the best of our knowledge, were not investigated previously in medical imaging.

We prove the benefits of the proposed fusion in regional cardiac motion abnormality detection following a standard issued by the American Heart Association [3], and comparing the results with ground truth classifications by radiologists. The evaluations in comparisons to the ground truth classifications showed that the proposed fusion brings a significant improvement of $3.8 \%$ in area-under-thecurve (AUC) accuracy. The experimental analysis was carried over 480 regional myocardial segments obtained from 30 subjects (20 normal and 10 abnormal). We evaluated the classifier ability of Shannon's differential entropies (SDE) of normalized radial distance and endocardial segment volume with and without fusion. The classifier ability of these features were measured using receiver operating characteristic (ROC) curves with the corresponding AUCs, and the

${ }^{1}$ Cardiac cine MRI is the most widely used MR acquisition protocol in clinical routine due to its low processing time and complexity over other MR acquisition methods. 


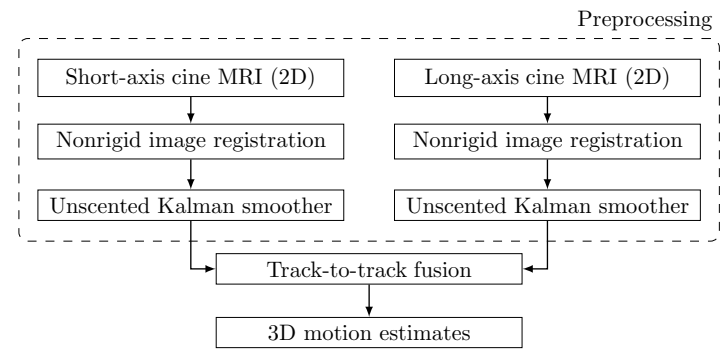

(a)

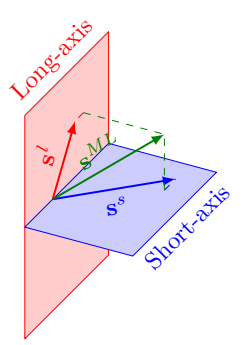

(b)

Fig. 1. (a) The proposed 3D motion estimation algorithm using $2 \mathrm{D}$ long and short-axis image fusion. (b) Illustration of a track-to-track maximum likelihood fusion $\mathbf{s}^{M L}$ using long- and short-axis state estimates $\mathbf{s}^{l}$ and $\mathbf{s}^{s}$.

Bhattacharyya distance metric [6]. We assessed the performance a via leave-onesubject-out approach. The proposed method yielded an AUC of 95.9\%, whereas the method without multiview fusion (i.e. using only short-axis images as is the case in [1]) yielded an AUC of $92.1 \%$.

\section{Track-to-Track Fusion}

Let $\mathbf{s}^{s}$ and $\mathbf{s}^{l}$ be the motion estimates obtained from, respectively, short- and long-axis images using UKS [12] and the registration algorithm [4. The state vectors $\mathbf{s}^{s}, \mathbf{s}^{l} \in\left\{[\bar{x} x \dot{x} \bar{y} \text { y } \dot{y} \bar{z} z \dot{z} \omega]^{T}\right\}$, where $(\mathrm{x}, \mathrm{y}, \mathrm{z})$ is a myocardial point on the $3 \mathrm{D}$ reference coordinate system corresponds to a pixel $(i, j)$ in the image coordinate system. $(x, y, z)$ is computed using a transformation matrix constructed based on the information from DICOM header. $[\dot{x} \dot{y} \dot{z}]$ is the velocity vector, $(\bar{x}, \bar{y}, \bar{z})$ the mean position of $(x, y, z)$ over a cardiac cycle, and $\omega$ the angular frequency.

Having obtained stałe estimates $\mathbf{s}^{s}$ and $\mathbf{s}^{l}$ corresponding to the same tissue from different views, we now have to combine these estimates. In this study, we propose a Maximum Likelihood (ML) criterion (Refer to Fig. 1)(b) for illustration) to compute a combined estimate $\mathbf{s}^{M L}$. We define the likelihood function as follows:

$$
\begin{aligned}
L(\mathbf{s}) & =-\ln p\left(\mathbf{s}^{s}, \mathbf{s}^{l} \mid \mathbf{s}\right) \\
& \propto\left(\left[\begin{array}{c}
\mathbf{s}^{s} \\
\mathbf{s}^{l}
\end{array}\right]-\left[\begin{array}{l}
I \\
I
\end{array}\right] \mathbf{s}\right)^{T} P^{-1}\left(\left[\begin{array}{l}
\mathbf{s}^{s} \\
\mathbf{s}^{l}
\end{array}\right]-\left[\begin{array}{l}
I \\
I
\end{array}\right] \mathbf{s}\right)
\end{aligned}
$$

where

$$
P=\left[\begin{array}{ll}
P^{s} & P^{s l} \\
P^{l s} & P^{l}
\end{array}\right]
$$

$P^{s}$ and $P^{l}$ are the covariances of $\mathbf{s}^{s}$ and $\mathbf{s}^{l}$, respectively, and $P^{s l}$ is the crosscovariance between $\mathbf{s}^{s}$ and $\mathbf{s}^{l} . I$ is an identity matrix. We compute the maximum 
likelihood solution,

$$
\mathbf{s}^{M L}=\arg \max _{\mathbf{s}} L(\mathbf{s}),
$$

by solving $\nabla_{\mathbf{s}} L(\mathbf{s})=0$. This yields:

$$
\mathbf{s}^{M L}=\left(\left[\begin{array}{ll}
I & I
\end{array}\right] P^{-1}\left[\begin{array}{l}
I \\
I
\end{array}\right]\right)^{-1}\left[\begin{array}{ll}
I & I
\end{array}\right] P^{-1}\left[\begin{array}{l}
\mathbf{s}^{s} \\
\mathbf{s}^{l}
\end{array}\right]
$$

Let $\mathcal{A}=P^{s}, \mathcal{B}=P^{s l}$ and $\mathcal{C}=P^{l}$. From inversion of a partitioned matrix, we have

$$
P^{-1}=\left[\begin{array}{cc}
\mathcal{A} & \mathcal{B} \\
\mathcal{B}^{T} & \mathcal{C}
\end{array}\right]^{-1}=\left[\begin{array}{cc}
\mathcal{E} & \mathcal{F} \\
\mathcal{F}^{T} & \mathcal{G}
\end{array}\right]
$$

where

$$
\begin{aligned}
\mathcal{E} & =\left(\mathcal{A}-\mathcal{B C}^{-1} \mathcal{B}^{T}\right)^{-1} \\
\mathcal{F} & =-\mathcal{E B C}^{-1} \\
\mathcal{G} & =\mathcal{C}^{-1}+\mathcal{C}^{-1} \mathcal{B}^{T} \mathcal{E} \mathcal{B C}
\end{aligned}
$$

Substituting for $P^{-1}$ in (4), we have

$$
\mathbf{s}^{M L}=\left(\mathcal{E}+\mathcal{F}^{T}+\mathcal{F}+\mathcal{G}\right)^{-1}\left(\mathcal{E}+\mathcal{F}^{T}\right) \mathbf{s}^{s}+\left(\mathcal{E}+\mathcal{F}^{T}+\mathcal{F}+\mathcal{G}\right)^{-1}(\mathcal{F}+\mathcal{G}) \mathbf{s}^{l}
$$

Substituting for $\mathcal{E}, \mathcal{F}$ and $\mathcal{G}$ from (6) - (6) and applying matrix inversion lemma (refer to Appendix for derivation details), we get

$$
\mathbf{s}^{M L}=\left(C-B^{T}\right)\left(\mathcal{A}+\mathcal{D}-\mathcal{B}-\mathcal{B}^{T}\right)^{-1} \mathbf{s}^{s}+(\mathcal{A}-\mathcal{B})\left(\mathcal{A}+\mathcal{C}-\mathcal{B}-\mathcal{B}^{T}\right)^{-1} \mathbf{s}^{l}
$$

Substituting $P^{s}, P^{s l}$ and $P^{l}$, we get

$$
\begin{aligned}
\mathbf{s}^{M L}=\left(P^{l}-P^{l s}\right)\left(P^{s}+P^{l}-P^{s l}-P^{l s}\right)^{-1} \mathbf{s}^{s} & \\
& +\left(P^{s}-P^{s l}\right)\left(P^{s}+P^{l}-P^{s l}-P^{l s}\right)^{-1} \mathbf{s}^{l}
\end{aligned}
$$

We assume that cross-covariance $P^{s l}, P^{l s}$ between short- and long-axis observations are zeros. Thus, we have

$$
\mathbf{s}^{M L}=P^{l}\left(P^{s}+P^{l}\right)^{-1} \mathbf{s}^{s}+P^{s}\left(P^{s}+P^{l}\right)^{-1} \mathbf{s}^{l}
$$

\section{Experiments}

The data contains $30 \times 3$ short-axis image datasets (i.e., apical, mid-cavity and basal), each consisting of 20 functional $2 \mathrm{D}$ images acquired from 20 normal and 10 abnormal hearts. The data were acquired on $1.5 \mathrm{~T}$ MRI scanners with fast imaging employing steady state acquisition (FIESTA) mode. In Fig. 2(a) and (b), we give a representative sample of the fusion results for end-diastolic and end-systolic phase of the cardiac cycle plotted against long-axis cine MR images. For each subject, three slices were respectively chosen from apical, mid-cavity 
and basal frames. In Fig. 2(c), (d) and (e), we give a representative sample of the segmentation results for apical, mid-cavity and basal frames. The frames were automatically segmented following the standard issued by the AHA [3], given anatomical landmarks on the first frame. The results of 480 myocardial segments were compared with a single ground truth classification. We classify a segment as abnormal if that segment is hypokinetic, akinetic or dyskinetic.

We used two independent criteria to measure the performance of each classifier features, namely, the ROC curves with corresponding AUCs [8], and Bhattacharyya measure [6] to assess the discriminative power of each classifier features. Furthermore, we assessed the performance of the proposed approach via a leave-one-subject-out method.

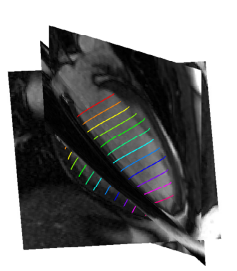

(a) End-diastole

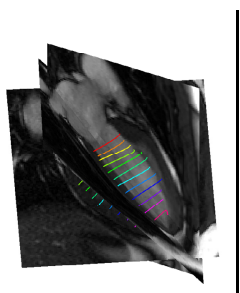

(b) End-systole

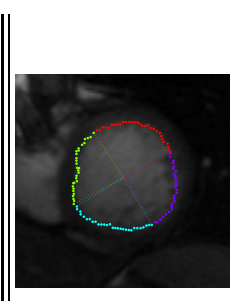

(c) Apical

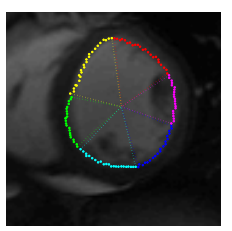

(d) Mid-cavity

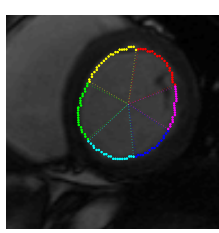

(e) Basal

Fig. 2. (a) and (b): Representative examples showing the obtained fusion estimates plotted against long-axis MR images; (c), (d) and (e): Representative examples of segmented myocardium using the proposed approach. Apical, mid-cavity and basal frames were segmented, respectively, into 4, 6 and 6 segments following the standard in 3 .

ROC, AUC and Bhattacharyya Measure: The ROC curves for classifier features SDEs of radial distance, segment area and segment volume are shown in Fig. 3. We used the same threshold for all segments and all slices. The ROC curves were obtained by varying such threshold. The AUCs corresponding to the ROC curves in Fig. 3] are reported in Table [1. The reported AUC values demonstrate that multiview fusion significantly improves the classifiers' ability in discriminating normal and abnormal heart motions.

We used the Bhattacharyya distance metric to evaluate the overlap between the distributions of classifier features over normal and abnormal motions. The Bhattacharyya metric [6] is given by $\mathbb{B}=\sqrt{1-\sum_{y \in \mathbb{R}} \sqrt{f_{N}(y) f_{A}(y)}}$, where $f_{N}$ and $f_{A}$ are the distributions over, respectively, normal and abnormal motions. The higher $\mathbb{B}$, the lesser the overlap and, therefore, the better the discriminative ability of the classifier. The Bhattacharyya distance metrics reported in Table 1 demonstrate that the multiview fusion significantly improve the discriminative ability of the classifier features in detecting abnormal heart motion.

Classification Performance: The evaluations of classification performance in terms of accuracy, sensitivity and specificity are given by accuracy $=\left(T_{P}+\right.$ 
Table 1. Comparison of the area under the ROC curve and Bhattacharyya distance metric for the methods with and without fusion (short-axis images only) [11.

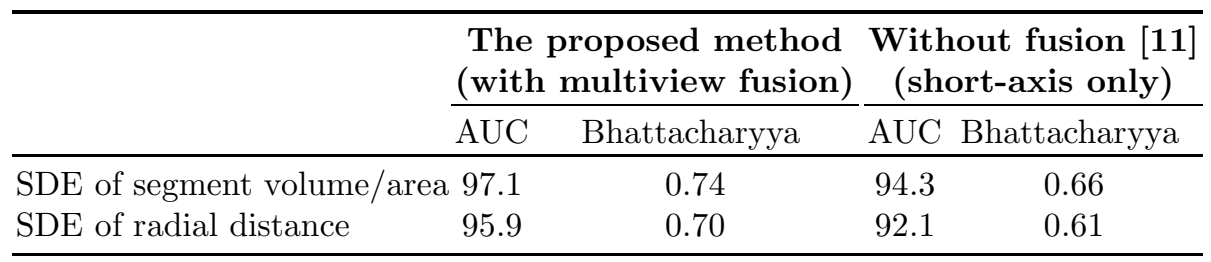

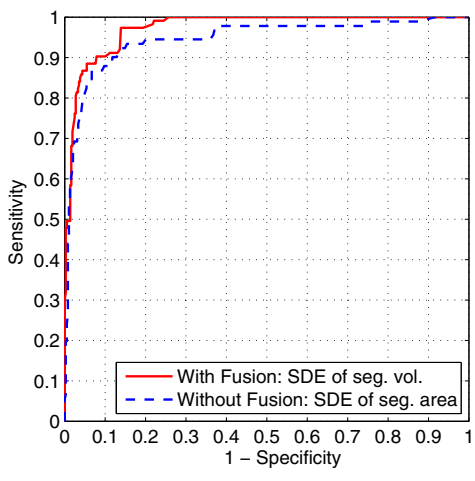

(a)

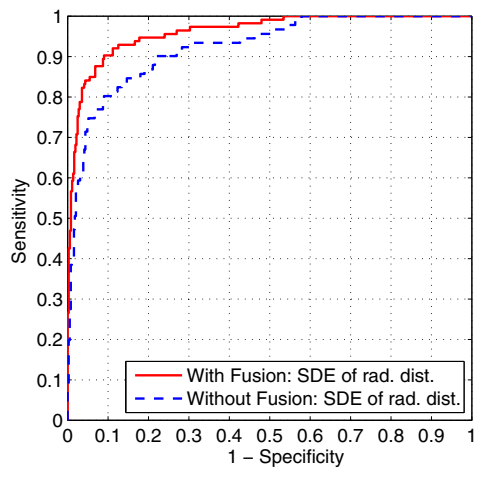

(b)

Fig. 3. Receiver operating characteristics of classifier features. The closer the curve to the left hand top corner, the better the classification performance.

$\left.T_{N}\right) /(P+N)$, specificity $=T_{N} / N$, sensitivity $=T_{P} / P$, where $T_{P}$ is true positives (number of segments correctly classified as "Abnormal") and $T_{N}$ true negatives (number of segments correctly classified as "Normal"). The number of "Abnormal" and "Normal" segments are $P$ and $N$, respectively. Table 2 compares the classification performance of correctly classified hearts with the proposed method and the method that uses only short-axis images [11, using a leaving-one-subject-out method. In this approach, a naive Bayes classifier algorithm [13] is constructed from the SDEs of the segment area and normalized radial distance. Fig. 4 shows the quadratic decision boundary for normal/abnormal classification with the proposed method (with the UKS), where blue circles represent the normal function and red triangles the abnormal. The decision boundaries were constructed separately for apical, mid-cavity and basal slices learning from the remaining 29 subjects. The overall classification accuracy for the proposed method with multiview fusion is equal to $91.9 \%$, with a sensitivity of $96.5 \%$ and specificity of $90.5 \%$. 
Table 2. The percentage of classification accuracy using a leaving-one-subject-out approach for the proposed track-to-track multiview fusion. The proposed method achieved an overall classification accuracy of $91.9 \%$.

\begin{tabular}{lrcc}
\hline & Accuracy $(\%)$ & Sensitivity $(\%)$ & Specificity (\%) \\
\hline Apex & 90.8 & 96.9 & 88.6 \\
Mid-cavity & 95.0 & 95.3 & 94.9 \\
Base & 89.4 & 97.4 & 87.3 \\
Overall & $\mathbf{9 1 . 9}$ & $\mathbf{9 6 . 5}$ & $\mathbf{9 0 . 5}$ \\
\hline
\end{tabular}

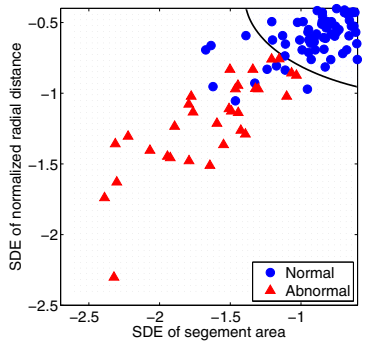

(a) Apical

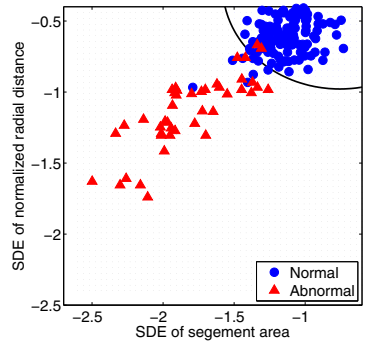

(b) Mid-cavity

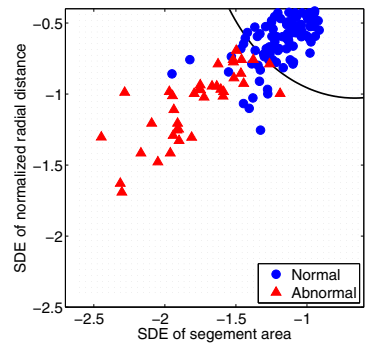

(c) Basal

Fig. 4. Decision boundary for normal and abnormal regional myocardial functions using a Bayesian classifier

\section{Conclusions}

This study investigated a track-to-track multiview fusion approach to $3 \mathrm{D} \mathrm{LV}$ motion estimation and regional abnormality detection. The proposed method uses several 2D cine MR image sequences, and yields state estimates in 3D space representing position and velocity information of myocardial points. A nonrigid image registration is used to obtain sequence of corresponding points and the UKS to track these points. Then, a track-to-track fusion method is proposed to combine UKS estimates from multiple images obtaining 3D state estimates. We show by an experimental evaluation that the proposed approach significantly improves the detection of regional abnormal motions in comparisons to previous approaches that use only the short-axis images.

\section{References}

1. Bar-Shalom, Y.: On the track-to-track correlation problem 26(2), 571-572 (April 1981)

2. Bistoquet, A., Oshinski, J., Skrinjar, O.: Left ventricular deformation recovery from cine MRI using an incompressible model. IEEE Trans. Med. Imag. 26(9), 1136-1153 (2007) 
3. Cerqueira, M.D., Weissman, N.J., Dilsizian, V., Jacobs, A.K., Kaul, S., Laskey, W.K., Pennell, D.J., Rumberger, J.A., Ryan, T., Verani, M.: Standardized myocardial segmentation and nomenclature for tomographic imaging of the heart: A statement for healthcare professionals from the cardiac imaging committee of the council on clinical cardiologyof the American Heart Association. Circulation 105(4), 539-542 (2002)

4. Chen, H.-M., Goela, A., Garvin, G.J., Li, S.: A Parameterization of Deformation Fields for Diffeomorphic Image Registration and Its Application to Myocardial Delineation. In: Jiang, T., Navab, N., Pluim, J.P.W., Viergever, M.A. (eds.) MICCAI 2010, Part I. LNCS, vol. 6361, pp. 340-348. Springer, Heidelberg (2010)

5. Chen, T., Wang, X., Chung, S., Metaxas, D., Axel, L.: Automated 3D motion tracking using gabor filter bank, robust point matching, and deformable models. IEEE Trans. Med. Imag. 29(1), 1-11 (2010)

6. Comaniciu, D., Ramesh, V., Meer, P.: Kernel-based object tracking. IEEE Trans. Pattern Anal. Mach. Intell. 25(5), 564-577 (2003)

7. Garcia-Barnes, J., Gil, D., Badiella, L., Hernandez-Sabate, A., Carreras, F., Pujades, S., Marti, E.: A normalized framework for the design of feature spaces assessing the left ventricular function. IEEE Trans. Med. Imag. 29(3), 733-745 (2010)

8. Hanley, J., McNeil, B.: The meaning and use of the area under a receiver operating characteristic (ROC) curve. Radiology 143(1), 29-36 (1982)

9. Lötjönen, J., Smutek, D., Kivistö, S., Lauerma, K.: Tracking Atria and Ventricles Simultaneously from Cardiac Short- and Long-Axis MR Images. In: Ellis, R.E., Peters, T.M. (eds.) MICCAI 2003. LNCS, vol. 2878, pp. 467-474. Springer, Heidelberg (2003)

10. Pan, L., Prince, J., Lima, J., Osman, N.: Fast tracking of cardiac motion using 3D-HARP. IEEE Trans. Biomed. Eng. 52(8), 1425-1435 (2005)

11. Punithakumar, K., Ben Ayed, I., Islam, A., Ross, I.G., Li, S.: Regional Heart Motion Abnormality Detection via Information Measures and Unscented Kalman Filtering. In: Jiang, T., Navab, N., Pluim, J.P.W., Viergever, M.A. (eds.) MICCAI 2010, Part I. LNCS, vol. 6361, pp. 409-417. Springer, Heidelberg (2010)

12. Sarkka, S.: Unscented Rauch-Tung-Striebel smoother. IEEE Trans. Automat. Contr. 53(3), 845-849 (2008)

13. Seber, G.A.F.: Multivariate Observations. John Wiley \& Sons, Inc., Hoboken (1984)

14. Spottiswoode, B., Zhong, X., Lorenz, C., Mayosi, B., Meintjes, E., Epstein, F.: 3D myocardial tissue tracking with slice followed cine DENSE MRI. J. Magn. Reson. Imag. 27(5), 1019-1027 (2008)

15. Suinesiaputra, A., Frangi, A., Kaandorp, T., Lamb, H., Bax, J., Reiber, J., Lelieveldt, B.: Automated detection of regional wall motion abnormalities based on a statistical model applied to multislice short-axis cardiac MR images. IEEE Trans. Med. Imag. 28(4), 595-607 (2009) 